\title{
Studies on Focal Adhesion Kinase in Human Breast Cancer Tissue
}

\author{
Kirat Kumar Ganguly ${ }^{1}$, Triparna Sen ${ }^{1}$, Syamsundar Mandal $^{2}$, Jaydip Biswas $^{3}$, Amitava Chatterjee ${ }^{1^{*}}$ \\ ${ }^{1}$ Department of Receptor Biology \& Tumor Metastasis, Chittaranjan National Cancer Institute, Kolkata, India; ${ }^{2}$ Department of Epi- \\ demiology \& Biostatistics, Chittaranjan National Cancer Institute, Kolkata, India; ${ }^{3}$ Department of Surgical Oncology, Chittaranjan \\ National Cancer Institute, Kolkata, India. \\ Email: *amitava_chatter@yahoo.co.in
}

Received November $26^{\text {th }}, 2011$; revised December $25^{\text {th }}, 2011$; accepted January $14^{\text {th }}, 2012$

\begin{abstract}
Aim: To study Expression and Phosphorylation status of Focal Adhesion Kinase (FAK) in Human Breast Cancer tissue. To study the relation of FAK with standard clinicopathological parameters of Human Breast Cancer. Methods: Tissue collection, Protein extraction, RNA isolation, Western Blot, Immunohistochemistry, RT-PCR, ELISA, Statistical analysis. Results: All the four techniques showed upregulated expression, phosphorylation (Tyr-397) and processing of FAK in human breast cancer tissue compared to the adjacent non-tumor tissue of the same patient. Upregulation of FAK was found to be increased parallely with the advancement of cancer. Localisation of FAK was found to be membrano-cytoplasmic. FAK is upregulated both in protein and mRNA level. Expression and phosphorylation of FAK is increased specifically in the tumor regions compared to the surrounding non-tumor region. Upregulation of FAK was frequently found in ER-positive and PR-positive but Her2/neu-negative breast cancer cases. Conclusion: FAK has crucial role in development and progression of human breast cancer. FAK may be considered as an indicator of human breast cancer progression. FAK processing may be considered as an indicator of invasive potential of breast cancer. FAK may be considered as a clinical indicator of human breast cancer development and progression.
\end{abstract}

Keywords: FAK; Human Breast Cancer

\section{Introduction}

Focal adhesion kinase (FAK) is a $125 \mathrm{kD}$ nonreceptor protein tyrosine kinase that localizes at the cellular adhesion points. It is a crucial mediator of signaling events between cells and cell surrounding extracellular matrix (ECM). FAK is ubiquitously expressed throughout development and well detectable in physiological adult tissues. FAK is mainly involved in integrin-signaling pathways, by binding with the $\beta 1$ subunit of Integrin [1-4]. Interaction of FAK with a number of signaling molecules situates it at a junction of signal transduction network and regulates cell migration [5-9] and apoptosis [10-17], cell proliferation [18-22] etc. In response to clustering of integrins due to ligand interaction, FAK is get activated by an autophosphorylation at Tyrosine-397 [23].

FAK imparts its activity both as a Kinase enzyme and an adaptor molecule $[24,25]$. During tumor development and cancer progression, it is thought that FAK have a double purpose both in promoting tumor cell adhesion during cell migration and in maintaining cell survival to inhibit apoptosis as a tumor develops anchorage-inde-

"Corresponding author. pendent growth properties.

The connection of FAK with breast cancer was first proposed by analyses with tissue homogenates showing that both FAK protein and mRNA were appreciably upregulated in invasive and metastatic breast tumor samples in comparison to matched normal tissues [26]. Latter on several studies showed crucial role of FAK in breast cancer. Importance of FAK in breast cancer biology was confirmed in experiments by depletion of FAK with antisense oligonucleotides causing an inhibition of cell migration, invasion and proliferation as well as induction of apoptosis [27-33]. Moreover, studies with xenograft models indicated role of FAK in subcutaneous tumor formation, in promotion of mammary tumor growth, in early phase of metastasis, in facilitation of angiogenic signal in mammary tumors etc $[30,34,35]$.

Studies by different groups with human tissue specimens have demonstrated specific up-regulation of FAK expression in high-grade sarcomas [36] and tumors from breast, colon, thyroid, ovarian, cervix, head-neck and esophagus [37]. Studies by several groups confirmed a pivotal role of FAK in tumorigenesis and metastasis [38]. These observations indicate towards the relevance FAK 
in human cancer.

Though a number of studies have demonstrated overexpression of FAK in transformed cells, primary and invasive carcinomas breast tissues $[39,40]$, the implication of these studies is still controversial. On the otherhand some groups claim that FAK is not overexpressed in human breast cancer cells or reduced FAK expression in liver metastases of colorectal adenocarcinoma, compared to the matched primary tumors [41]. Number of early analyses was dependent largely on tissue extraction methods to demonstrate FAK mRNA and protein overexpression, which may give false result due to contaminants of surrounding normal cell in tumor extract. Thus, the significance of FAK expression in human tumors remains to be resolved.

Relation of FAK with other clinicopathological parameter, including TNM stages or well accepted markers like Her2/neu, ER, PR, was also studied by some groups $[42,43]$ Her2/neu (Her2) is a receptor tyrosine kinase belongs to EGF receptor (EGFR)/ErbB family. In physiological tissue, Her2 heterodimerizes with other ErbB family members after that protein binds corresponding ligand. Overexpression of Her2 results in constitutive, ligand-independent activation of tyrosine kinase signaling which is found in a number of human breast cancers. In those cases it is associated with a more aggressive cancer phenotype and poorer prognosis [44]. Estrogen Receptor (ER) is the intracellular receptor of Estrogen. ER is overexpressed in $70 \%$ of breast cancer cases [45]. Another marker protein progesterone Receptor (PR), receptor of the hormone Progesterone, also reflects the status of breast cancer [46]. Though the connection of these parameters with FAK expression was studied, but the overall relations remain inconclusive and need more studies to get the precise role and correlation of FAK with breast cancer.

Considering all these factors, we have studied both expression and phosphorylation status of FAK in a population of breast cancer patients of different stages and have tried to strengthen the relation of FAK expression with human breast cancer.

\section{Materials and Methods}

\subsection{Materials}

Protease Inhibitor Cocktail Tablets (complete, mini, EDTAfree) were purchased from Roche, Germany. Primary antibodies (anti-FAK, anti-p-FAK-Tyr-397 and anti- $\beta$-tubulin) antibodies were purchased from Santa Cruz, USA. Biotinylated second-antibodies, SuperSignal West Femto Maximum Sensitivity Substrate were purchased from Thermo Scientific, Rockford, USA. Avidin-biotinylated peroxidase complex reagent (vectastain Rabbit $\mathrm{ABC}$ kit) was purchased from vector laboratories, Burlingame, CA).
DAB substrate and stable peroxidase substrate buffer were purchased from Pierce Biotechnology, USA. Primers were synthesized by Operon, Germany. Trizol Reagent was purchased from Invitrogen, Retro-script (RTPCR Kit) was purchased from Ambion, USA. Immobilon-P Membrane, (PVDF), was purchased from Millipore, USA.

\subsection{Patient}

Our present study involves 37 breast cancer female patients. They were referred to Chittaranjan National Cancer Institute, India because of clinical breast lump, suspicious mammographic finding or a breast symptom (e.g. pain, nipple discharge) between 2008 and 2010. Women willing to participate in the project were interviewed and examined by a trained study nurse before any diagonostic procedures. The participation rate of patients with diagonosed breast cancer was $98 \%$. Thus the patient series represents unselected typical breast cancer cases of different stages from the institutional hospital catchment area. After surgical treatment, the patients were offered adjuvant chemotherapy and/or hormonal therapy or radiotherapy, depending on the mode of the surgery, the patients' menopausal status, and the stage of the disease, according to the national guidelines. In brief postoperative radiotherapy was given to all patients treated with breast-conserving and to all patients with axillary node positive $(\mathrm{pN}+)$ status irrespective of the mode of surgery. All menopausal patients with $\mathrm{pN}+$ status and some with axillary node negative $(\mathrm{pN}-)$ status presenting with other adverse prognostic factors such as estrogen receptor(ER)/ progesterone receptor (PR) negative or poorly differentiated tumor, were given adjuvant chemotherapy, intravenous cyclophosphamide, mitoxantrone, methotrexate and 5-flurouracil) for six cycles. All post menopausal women with ER or PR positive tumors were adjuvently treated with either tamoxifen or toremifene for 3 years within a clinical study protocol. Thus, within a stage, the postoperative treatment was rather uniform, with only a few exceptions due to concurrent conditions. Stage was assessed by using the UICC classification. Patients with noninvasive carcinomas, a previous history of breast cancer, metastatic disease (stage-IV), or insufficient tumor material was excluded from the present study. Thus 37 patients with sufficient primary tumors and complete clinical histories were available for the present study. The mean age of the patients was 59.2 years (median 56.8 years; range, 23.3 - 91.6 years). The mean follow up time was 55.0 months (median 57.5 months; range, 1.2 115.1 months). The clinicopathological data of the patients are summarized in Table 1.

\subsection{Collection of Tissues Samples}

Tissue specimens from tumor and respective adjacent 
Table 1. Clinico-pathological parameters of the study population.

\begin{tabular}{|c|c|}
\hline Parameter & Number (\%) \\
\hline Total Number of subjects & 37 \\
\hline \multicolumn{2}{|l|}{ Age } \\
\hline Below 45 years & $19(51.4 \%)$ \\
\hline 45 years and above & $18(48.6 \%)$ \\
\hline \multicolumn{2}{|l|}{ Stage at diagnosis } \\
\hline Early (IA, IIA \& IIB) & $16(43.2 \%)$ \\
\hline Advanced (IIIA, IIIB \& IIIC) & $21(56.8 \%)$ \\
\hline \multicolumn{2}{|l|}{ Estrogen receptor } \\
\hline Positive & $13(35.1 \%)$ \\
\hline Negative & $24(64.9 \%)$ \\
\hline \multicolumn{2}{|l|}{ Progesterone receptor } \\
\hline Positive & $15(40.5 \%)$ \\
\hline Negative & $22(59.5 \%)$ \\
\hline \multicolumn{2}{|l|}{ HER-2/neu } \\
\hline Positive & $15(40.5 \%)$ \\
\hline Negative & $22(59.5 \%)$ \\
\hline \multicolumn{2}{|l|}{ FAK (by Western blot) ${ }^{*}$} \\
\hline High & $20(54.0 \%)$ \\
\hline Not High & $17(46.0 \%)$ \\
\hline \multicolumn{2}{|l|}{ p-FAK (by Western blot) ${ }^{*}$} \\
\hline High & $24(64.9 \%)$ \\
\hline Not High & $13(35.1 \%)$ \\
\hline \multicolumn{2}{|l|}{ FAK (by IHC) ${ }^{\#}$} \\
\hline High & $19(51.3 \%)$ \\
\hline Not High & $18(48.7 \%)$ \\
\hline \multicolumn{2}{|l|}{ p-FAK (by IHC) ${ }^{\#}$} \\
\hline High & $20(54.0 \%)$ \\
\hline Not High & $17(46.0 \%)$ \\
\hline
\end{tabular}

"Western Blot: FAK high denotes difference in FAK expression between Tumor and Normal $\geq$ mean difference i.e. 50 (expression of FAK was measured densitometrically). FAK not high denotes difference in FAK expression between Tumor and Normal $\leq$ mean difference i.e. 50 (expression of FAK was measured densitometrically). " Immunohistochemistry: FAK high $=3$ and 4 score. FAK not high $=0,1,2$ score.

non-tumor breast tissues of the same patient were collected from the operation theater during surgery. Tissues were stored at $-80^{\circ} \mathrm{C}$ and used for the further experiments.

\subsection{Protein Extraction from Tissue}

Tissues of tumor samples and respective adjacent nontumor breast tissues were collected, extracted with cell extraction buffer (Tris: $37.7 \mathrm{mM}, \mathrm{NaCl}: 75 \mathrm{mM}$, Triton $\mathrm{X}-100$ : $0.5 \%$, protease inhibitor cocktail and $\mathrm{pH}$ adjusted to 7.5) and the protein content of the extracts were estimated by Lowry's method.

\subsection{Western Blot}

Equal amount of protein $(50 \mu \mathrm{g}$ each) was taken and heated with 0.1 volumes $\beta$-mercaptoethanol for $5-8 \mathrm{mi}$ nutes at $80^{\circ} \mathrm{C}-90^{\circ} \mathrm{C}$, then subjected to electrophoresis on SDS-PAGE. The proteins were electrophoretically transferred on PVDF membranes. The membranes were blocked with $1 \%$ BSA and subsequently washed $\times 3$ with PBST. The membranes were reacted with anti-FAK
(A-17) antibody, anti-FAK (Tyr-397)-R antibody and anti- $\beta$-tubulin (G-8) antibody at 1:2000 dilution with $1 \%$ BSA each and kept at $37^{\circ} \mathrm{C}$ for $1 \mathrm{Hr} 30$ mins and subsequently washed thrice with PBST. The blots were developed using respective horse redish peroxidase (HRP) coupled second antibodies at 1:200,000 dilution and kept at $37^{\circ} \mathrm{C}$ for $1 \mathrm{Hr} 30 \mathrm{mins}$, the blots were then thoroughly washed 6 times with TBST. Bands were visualized using protocol given with SuperSignal substrate.

\subsection{RNA Isolation}

Both adjacent non-tumor and respective tumor tissues were collected, homogenized in $1 \mathrm{ml}$ of Trizol reagent per $50 \mu \mathrm{g}$ of tissue using a power homogenizer. Homogenized samples were incubated for 5 minutes at room temperature (RT). Then $200 \mu \mathrm{l}$ of chloroform was added to each sample, vigorously shaken for 15 seconds and incubated at RT for $2-3$ minutes. Centrifuged at $12.000 \times \mathrm{g}$ for 15 minutes at $4{ }^{\circ} \mathrm{C}$. Aqueous phase was isolated and mixed with $0.5 \mathrm{ml}$ of isopropyl alcohol, incubated for 10 minutes at RT, centrifuged at $12000 \times \mathrm{g}$ for 10 minutes at $4^{\circ} \mathrm{C}$. Supernatant removed and RNA pellet was washed with $75 \%$ ethanol and air dried and dissolved in preheated RNAse free water. Concentration and purity of RNA was estimated by standard protocol.

\subsection{Semi-Quantitative RT-PCR}

The 2 steps RT-PCR (Retroscript, Ambion, USA) was done with equal amounts of total RNA, using specific primers for PCR (FAK and GAPDH). $20 \mu 1$ of each PCR products were run on a $2 \%$ agarose gel and bands visualized under UV. Glyceraldehyde phosphate dehydrogenase (GAPDH) primers were used as control to normalize for mRNA equal loading. The primer sequences and PCR cycles/conditions for each primer are tabulated below.

\section{Primer sequences:}

hFAK:

5'-CGCTGGCTGGAAAAAGAGGAA-3'(forward), 5'-TCGGTGGGTGCTGGCTGGTAGG-3'(reverse).

\section{GAPDH:}

5'-CGGAGTCAACGGATTTGGTCGTAT-3'(forward), 5'-AGCCTTCTCCAT GGTGGTGAAGAC-3'(reverse).

PCR conditions:

$\left(94^{\circ} \mathrm{C}-30 \operatorname{secs}, 60^{\circ} \mathrm{C}-30 \operatorname{secs}, 72^{\circ} \mathrm{C}-90 \operatorname{secs}\right)$.

\subsection{Enzyme-Linked Immunosorbent Assay (ELISA)}

Equal amount of protein $(5 \mu \mathrm{g}$ each) was taken and coated in a 96 well flat bottom, sterile culture plate and subjected to ELISA with anti-FAK (A-17) antibody, antiFAK (Tyr-397)-R antibody using HRP-coupled secondary antibody and TMB/ $\mathrm{H}_{2} \mathrm{O}_{2}$ as substrate for enzymatic reaction. 


\subsection{Quantification of the Results}

Bands Western blots and RT-PCR were quantified using Image J Launcher (version 1.4.3.67).

\subsection{Histology}

Both normal and tumor samples were fixed in 10\% neutral buffered formalin, processed routinely and embedded in paraffin. The histologic diagnosis was confirmed by reviewing one to four original sections of the primary tumor. All tumors were simultaneously reevaluated for histologic type and grade by one senior pathologist, who was unaware of the clinical data. The most representative blocks were selected for cutting into new $5 \mu \mathrm{m}$-thick sections for immunohistochemical analyses.

\subsection{Immunohistochemistry}

The immunostaining of FAK, p-FAK were demonstrated using sequenza Immunostaining center (Shandon Scientific Limited, Astmoor, UK). In brief, the sections were de-parafinized in xylene, re-hydrated with graded EtOH, and distilled water. For better antigen retrieval, the samples were boiled three times for 15 minutes in boiling water in citrate buffer ( $10 \mathrm{mM}, \mathrm{pH} 6)$. Endogenous peroxidases were blocked by $0.3 \%$ hydrogen peroxidase treatment for 5 minutes. The samples were washed with PBS (pH 7.2) and incubated in $2 \%$ normal bovine serum for 35 minutes to prevent nonspecific antigen binding followed by incubation with The primary antibody for FAK (A-17) or p-FAK (Tyr-397)-R at a working dilution of 1:500 for overnight at $4^{\circ} \mathrm{C}$. Before applying the secondary antibody, the samples were washed twice with PBS. The slides were then incubated with the biotinylated secondary antibody followed by a wash and 50 minutes incubation in an avidin-biotinylated peroxidase complex reagent. Expressions were visualized with a 5-minute diaminobenzidin tetrahydrochloride treatment. The slides were counterstained with Mayer's hematoxylin, dehydrated and mounted with DePex. A routinely processed breast cancer section without the primary antibody served as negative control at each staining series.

\subsection{Scoring of Immunoreactivity}

Expression of FAK or p-FAK (Tyr-397) was recorded separately for tumor and normal cells. A pathologist scored each tissue section for FAK and p-FAK expression based on a scoring system which measured intensity (0-none; 1-borderline; 2-weak; 3-moderate; 4strong), and cellular localization (nucleus, cytoplasm, membrane, or membrano cytoplasmic). To define the relation statistically, we classified FAK and p-FAK expression as high (3+ or $4+$ intensity) and not high $(0,1+$, and $2+$ intensity).

\subsection{Statistical Methods}

Statistical analyses had been performed using EPI Info ${ }^{\mathrm{TM}}$ 3.5.3 developed by the Centres for Disease Control and Prevention (CDC). Both univarite analysis and multivariate analysis were used to analyze the data. Under univarite analysis Chi-square test and odd ratios with 95\% confidence interval had been calculated and corresponding p-values were found. Under multivariate analysis multivariate analysis Logistic Regression method had been used to estimate the odds ratios with 95\% confidence interval.

\section{Results}

\subsection{Immunoblot Analysis Showed Expression and Phosphorylation (Tyr 397) of FAK Is Increased in Tumor Samples Compared to the Non-Tumor Counterpart}

Western blot analysis revealed that the expressions of pp125 along with the cleaved products of FAK were increased in tumor samples compared to the non-tumor counterpart. Phosphorylation of FAK at Tyr 397 was also found to be increased in the tumor samples. For comparison of relative amount of FAK or p-FAK, we considered only the band of $125 \mathrm{kD}$. The differences between Tumor and nontumor samples were found to become notably greater with the advancement of clinical stages (Stage I to Stage III C) of cancer. In Stage I, FAK and p-FAK expression was respectively 1.13 and 1.17 fold higher in tumor compared to the adjacent non-tumor samples. Whereas, In Stage IIIC specimens, FAK and p-FAK expression was found to be 2.70 and 1.76 folds upregulated in tumor samples compared to the adjacent non-tumor samples, respectively. For statistical analysis we considered the difference of FAK expression, between non-tumor and tumor samples, for each patient. This value indicated the overexpression of FAK. We considered the values above the mean difference as high expression of FAK. In our study $54.0 \%$ of total number of patients showed high expression of FAK and 64.9\% cases showed high phosphorylation FAK. Statistical analysis revealed, risk of increased expression and phosphorylation of FAK was found more in ER and PR-positive cases but Her2/neu-negative cases. It indicates direct relation of FAK with ER, PR but inverse relationship with Her-2/neu. Interestingly upregulation of FAK was found in lower age-group (Below 45 years) patients (Figure 1, Tables 2(a) and (b)).

\subsection{Expression of FAK and P-FAK Was Also Found to Be Increased When Checked by ELISA}

ELISA also showed the same pattern of increase in FAK 


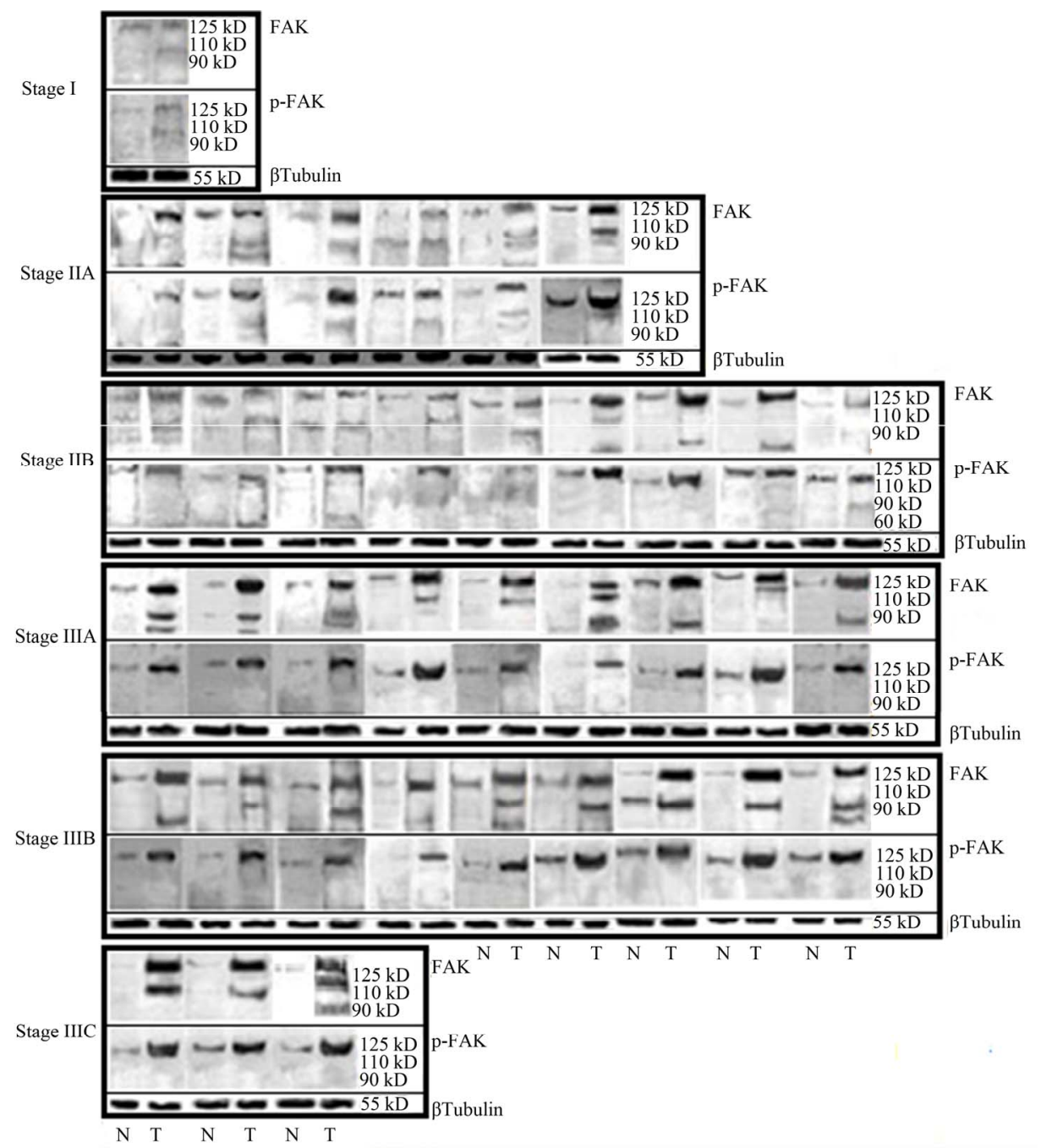

Figure 1. FAK expression and phosphorylation (Tyr 397) is upregulated in human breast cancer: matched pairs of tumor and non-tumor specimens from the same patient were analysed for their FAK expression \& phosphorylation by Western blot. $N$ stands for Non-tumor, and $T$ stands for tumour sample. Total number of samples was grouped depending on the clinical stages (Stage IIA, IIB, IIIA, IIIB, IIIC). $\beta$-Tubulin (55 kD) as loading control.

Table 2. (a) Relation between FAK and clinicopathological parameters (data taken from western blot analysis); (b) Relation between p-FAK and clinicopathological parameters (data taken from western blot analysis)

(a)

\begin{tabular}{|c|c|c|c|c|c|c|}
\hline \multirow{2}{*}{ Parameters } & \multirow{2}{*}{ FAK High } & \multirow{2}{*}{ FAK Not High } & \multicolumn{2}{|l|}{ Univariate } & \multicolumn{2}{|l|}{ Multivariate } \\
\hline & & & Odds ratio & p-value & Odds ratio & p-value \\
\hline \multicolumn{7}{|l|}{ Age } \\
\hline Below 45 years & 13 & 06 & $3.40(1.72,17.05)$ & 0.07 & $0.90(0.06,12.43)$ & 0.94 \\
\hline 45 years and above & 07 & 11 & & & & \\
\hline \multicolumn{7}{|l|}{ Stage } \\
\hline Early & 02 & 14 & $42.00(4.78,533.29)$ & 0.00001 & $45.34(2.62,78.51)$ & 0.0087 \\
\hline Advanced & 18 & 03 & & & & \\
\hline \multicolumn{7}{|l|}{ Estrogen receptor } \\
\hline Negative & 10 & 14 & $4.67(1.83,29.30)$ & 0.03 & $1.04(1.01,15.45)$ & 0.097 \\
\hline Positive & 10 & 03 & & & & \\
\hline \multicolumn{7}{|c|}{ Progesterone receptor } \\
\hline Negative & 9 & 13 & $3.97(1.78,21.77)$ & 0.05 & $10.93(1.52,22.86)$ & 0.012 \\
\hline Positive & 11 & 4 & & & & \\
\hline \multicolumn{7}{|l|}{ HER-2/neu } \\
\hline Negative & 15 & 7 & $4.29(1.86,22.87)$ & 0.04 & $3.45(1.29,39.84)$ & 0.032 \\
\hline Positive & 5 & 10 & & & & \\
\hline
\end{tabular}


(b)

\begin{tabular}{|c|c|c|c|c|c|c|}
\hline \multirow{2}{*}{ Parameters } & \multirow{2}{*}{ FAK High } & \multirow{2}{*}{ FAK Not High } & \multicolumn{2}{|l|}{ Univariate } & \multicolumn{2}{|l|}{ Multivariate } \\
\hline & & & Odds ratio & p-value & Odds ratio & p-value \\
\hline \multicolumn{7}{|l|}{ Age } \\
\hline Below 45 years & 06 & 13 & $3.40(1.72,17.05)$ & 0.064 & $0.48(0.08,2.80)$ & 0.41 \\
\hline 45 years and above & 07 & 11 & & & & \\
\hline \multicolumn{7}{|l|}{ Stage } \\
\hline Early & 04 & 12 & $2.25(1.44,12.11)$ & 0.025 & $1.32(1.17,10.18)$ & 0.078 \\
\hline Advanced & 09 & 12 & & & & \\
\hline \multicolumn{7}{|l|}{ Estrogen receptor } \\
\hline Negative & 06 & 18 & $3.50(1.67,19.29)$ & 0.07 & $1.29(1.14,11.45)$ & 0.081 \\
\hline Positive & 07 & 06 & & & & \\
\hline \multicolumn{7}{|c|}{ Progesterone receptor } \\
\hline Negative & 05 & 17 & $3.89(1.76,21.28)$ & 0.06 & $4.58(1.49,12.92)$ & 0.018 \\
\hline Positive & 08 & 07 & & & & \\
\hline \multicolumn{7}{|l|}{ HER-2/neu } \\
\hline Negative & 10 & 12 & $3.33(1.60,20.62)$ & 0.01 & $4.55(1.62,13.16)$ & 0.013 \\
\hline Positive & 03 & 12 & & & & \\
\hline
\end{tabular}

FAK high: difference in FAK expression between Tumor and Normal $\geq$ mean difference i.e. 50 (expression of FAK was measured densitometrically); FAK not high: difference in FAK expression between Tumor and Normal $\leq$ mean difference i.e. 50 (expression of FAK was measured densitometrically).

expression and phosphorylation (Tyr 397) like Western blot analysis. In ELISA, we found average FAK expression increased 1.23 folds in tumor tissue of stage I, but 4.22 folds increase in FAK expression in stage IIIC specimens compared to the corresponding adjacent nontumor specimens. Whereas, in tumor samples p-FAK increased 1.145 folds in stage I samples and 4.15 folds in case of stage IIIC specimens corresponding adjacent nontumor specimens (Figure 2).

\subsection{FAK mRNa Expression Was Increased in Tumor Samples}

RT-PCR revealed that FAK m-RNA expression is increased in Tumors with respect to adjacent non-tumor specimens. With the progression of clinical stages, mRNA expression was found to be increased in Tumor samples compared to the non-tumor counterpart. The average FAK mRNA expression were increased 1.02 folds in stage I tumor specimens compared to the adjacent nontumor samples. Whereas the increase was found 3.66 folds in case of stage IIIC samples (Figure 3).

\subsection{Immunohistochemistry Showed Upregulation of FAK and P-FAK Specifically in Tumor Regions Compared to the Normal Tissue Regions}

Immunohistochemical analysis of FAK expression and phosphorylation (Tyr-397) in tumours vs correspo adjacent non-tumor breast tissue showed comparable results like the Western blot analysis. In tissue sections FAK and p-FAK (Tyr 397) was found to be increased specifically in transformed cell clusters compared to the sur- rounding normal tissue regions. FAK and p-FAK was mainly found to be localized in the membrane and cytoplasm of the cells. Some of the adjacent non-tumor cells showed very weak expression of FAK and p-FAK which was found to be upregulated in the transformed cells. The expressions were gradually increased with the progression of cancer stages. Immunohistochemistry showed
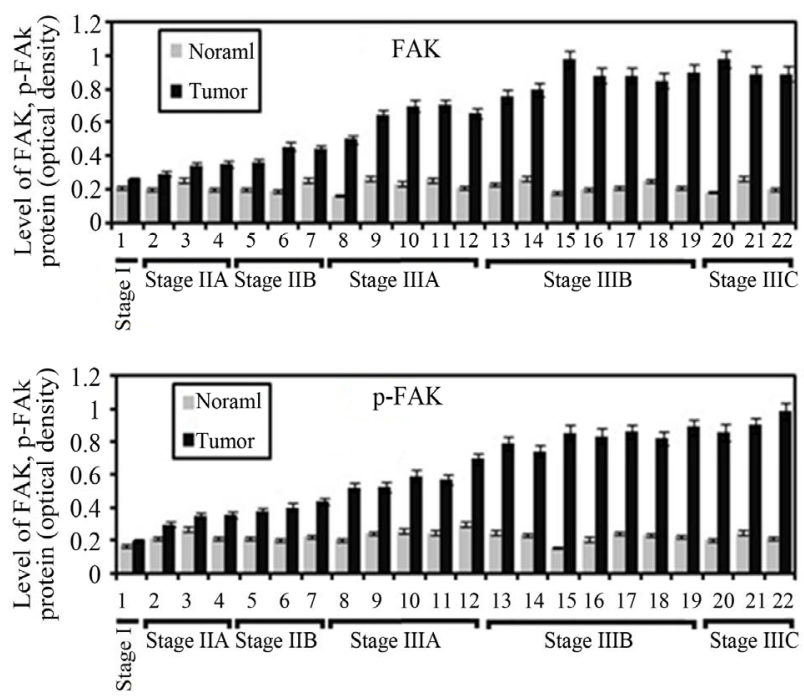

Figure 2. Upregulation of FAK expression and phosphorylation (Tyr 397) in breast cancer measured by ELISA: matched pairs of Tumor and non-tumor specimens from the same patient were analysed for their FAK expression and phosphorylation by ELISA. $N$ stands for Non-tumor, and $T$ stands for tumour sample. Total numbers of samples were grouped depending on the clinical stages. Stage Isample 1, IIA-sample 2-4, IIB-sample 5-7, IIIA-sample 8-12, IIIB - sample 13-19, IIIC-sample 20-22). 

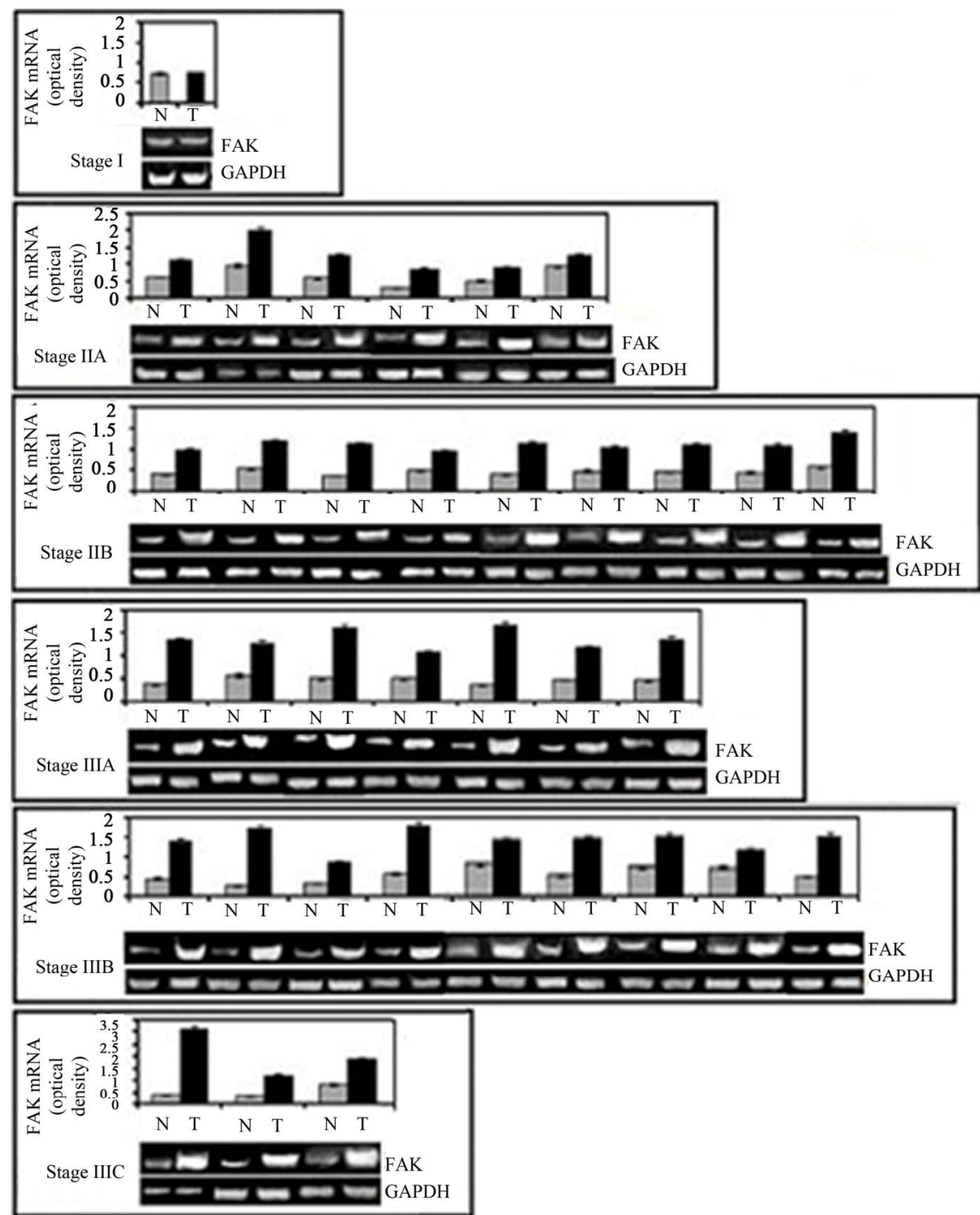

Figure 3. FAK is overexpressed in mRNA level in human breast cancer. RT-PCR analysis of FAK mRNA (475 bp) expression in matched pairs of non-Tumor and Tumor breast tissue using FAK-specific primer. GAPDH mRNA was studies as an internal control (454 bp). $\mathrm{N}$ stands for Non-tumor, and $\mathrm{T}$ stands for tumor sample. The samples were grouped according to the clinical staging.

$51.3 \%$ of our total study populations have FAK overexpression and $54.0 \%$ exhibited higher phosphorylation of FAK (Figure 4, Tables 3(a) and (b)).

\section{Discussion}

Focal adhesion kinase (FAK), the non-receptor protein tyrosine kinase is the upstream molecule of Integrin mediated signaling. FAK is involved in regulation of different cellular processes in physiological condition which are also found to be associated with the development of cancer, like cell adhesion [47,48], migration [5-9], invasion [49,50], survival [10-17], proliferation [18-21], differentiation [51]. Breast cancer is the second leading cause of death due to cancer among woman. In 1995 Owen et al. for the first time showed higher level of FAK protein and mRNA in metastatic breast cancer tissue samples [26]. They suggested that FAK may be a marker of invasive potential of tumor. After that a number of studies have shown correlation of FAK with the development and progression of different types of human cancers [52,53]. Several studies further confirmed elevated expression of FAK in breast cancer [38,43,44,54]. Latter it was also proposed that FAK overexpression is not only restricted to the higher invasive types of cancer but also associated with the early events of tumorigenesis [55]. Studies indicated association of FAK expression with the indicators of poor prognosis and with progression of metastasis $[38,40,43]$. In contrast, some studies have shown inter-relationship between FAK expression and cancer progression, where progression of metastasis 
was reported to be linked with weak FAK expression [56]. With these variations in background we have tried to study status of FAK expression and phosphorylation in a population of patients with different stages of breast cancer, and tried to increase the knowledge of relation between FAK with breast cancer.

Early reports showed Integrin clustering, after specific ligand binding, results in autophosphorylation of FAK at Tyrosine-397 both in attachment dependent and independent cells (57). Since this is the first event of FAK stimulation, this phosphorylation (Tyr397) is considered as the indicator of FAK activation. In that way tumor samples in our study indicated elevated FAK activity with the development and progression of breast cancer. Madan et al. correlated increased FAK phosphorylation (Tyr-397) with the stage of malignant transformation, not with the development of tumor metastasis. On the other hand studies have indicated this phosphorylation as crucial regulator of cell migration [57,58]. In another study, our group has found Tyr-397 phosphorylation of FAK is increased when invasive breast cancer cell line MDAMB-231 and MCF-7 were treated with Fibronectin (FN). In the same condition cell migration was also found to be accelerated. FN is over-expressed in tumor tissue, which may be the cause of increased FAK activity.

Cleavage of FAK to lower molecular weight residues have shown to be connected to cell migration. Brattain et al. explains that during cell migration, attachment of the leading edge of the cell is coupled with the building of focal adhesion complexes, whereas detachment at the rear-end of the cell results in the dismantling of focal adhesion complexes and the cleavage of FAK. In our previous study on FN treated model of MDA-MB-231 and MCF-7, we found increased FAK processing simultaneously with higher cell migration and invasion. Cleavage of FAK is proposed to be linked with the dismantling of focal adhesions [59]. Conclusively, Brattain et al. proposed that cleavage of FAK is essential for pathological cell motility, like cancer metastasis. This observation can explain our result of increased FAK cleavage products in Tumor samples compared to the adjacent non-tumor samples. The processing was found to be increased parrallely with the cancer stage progression, which may be as a result of increased migration \& invasion of the transformed cells to surrounding tissue, nodes or distant organs. FAK is essential for several activities both in physiological and pathological conditions. So, a basal level of FAK is produced in normal tissue. Increase in FAK expression and phosphorylation (Tyr 397) in tumor specimens indicates the relevance of FAK in tumor development and differentiation. Our previous study also showed over expression and phosphorylation of FAK in FN treated breast cancer cell line. Whereas, depletion of FAK, under the same condition, was found to decrease cell migration along with the decrease in tow important regulators of cell migration and invasion, MMP-9 and MMP-2. Again, FAK was established as antiapoptotic and an important survival signal by several groups [10-17]. Depletion of FAK results in cell death [60]. The process of apoptosis is ceased in tumor cells. So, the elevated expressions of FAK in our observation are also corroborating with nature of FAK and accept the previous reports on overexpression of FAK in breast cancer $[40,42,43,54]$.

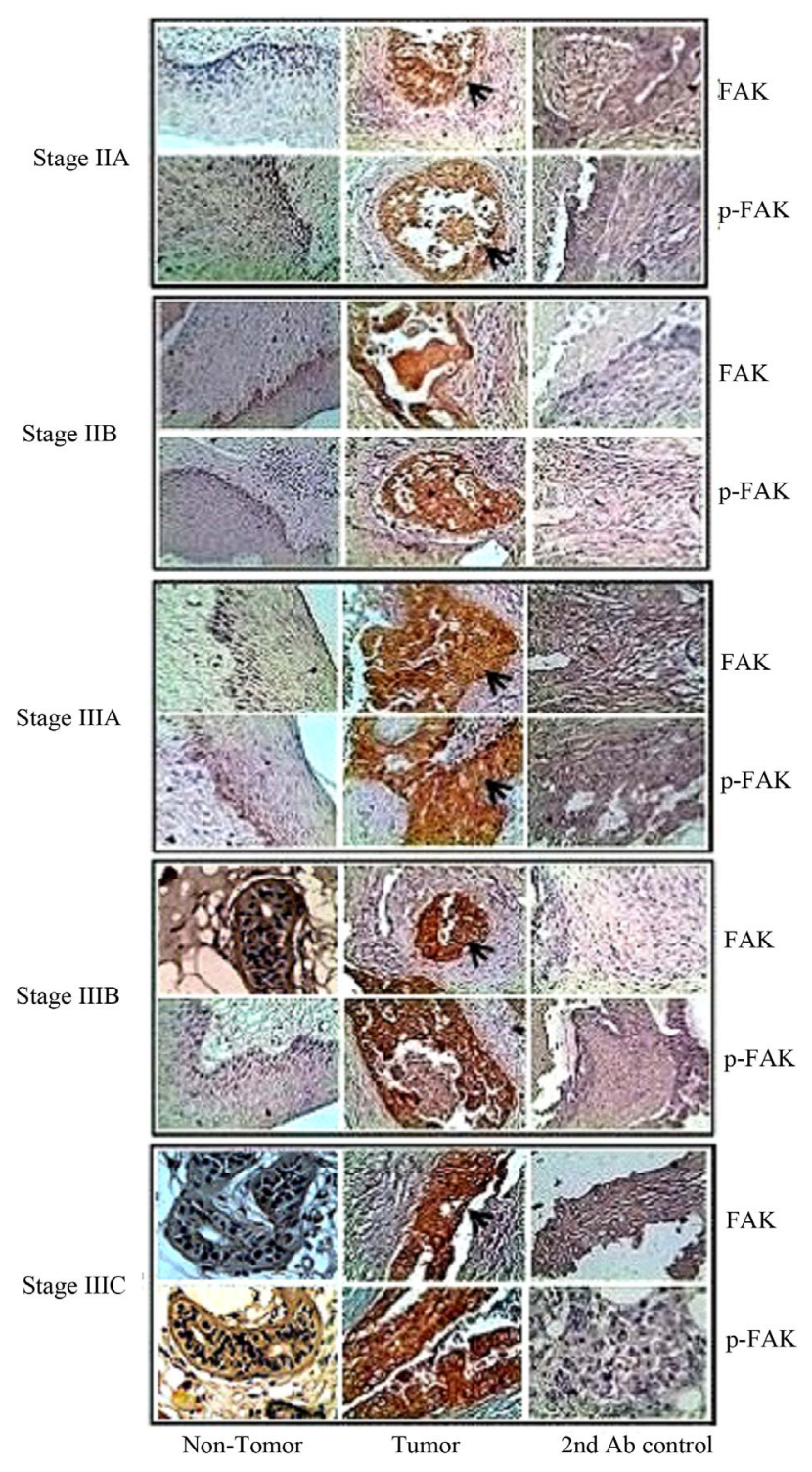

Figure 4. Immunohistochemistry with anti-FAK and anti-pFAK antibody in breast cancer tissue sections: Immunohistochemical analysis of FAK and p-FAK in breast tissue of the same patient of different clinical stages (Stage IIA, IIB, IIIA, IIIB, IIIC). Expression and phosphorylation of FAK detected by brown stain of DAB. Second Antibody control ( $2^{\text {nd }}$ Ab Ctrl) (without FAK \&/or p-FAK primary antibody) was done to exclude the possibility of non-specific attachment of second antibody. 
Table 3. (a) Relation between FAK and clinicopathological parameters (data taken from IHC analysis); (b) Relation between p-FAK and clinicopathological parameters (data taken from IHC analysis).

(a)

\begin{tabular}{|c|c|c|c|c|c|c|}
\hline \multirow{2}{*}{ Parameters } & \multirow{2}{*}{ FAK High } & \multirow{2}{*}{ FAK Not High } & \multicolumn{2}{|l|}{ Univariate } & \multicolumn{2}{|l|}{ Multivariate } \\
\hline & & & Odds ratio & p-value & Odds ratio & p-value \\
\hline \multicolumn{7}{|l|}{ Age } \\
\hline Below 45 years & 13 & 06 & $5.63(1.11,31.02)$ & 0.013 & $10.16(1.55$ & 0.015 \\
\hline 45 years and above & 05 & 13 & & & $66.39)$ & \\
\hline \multicolumn{7}{|l|}{ Stage } \\
\hline Early & 0 & 16 & -- & -- & -- & -- \\
\hline Advanced & 18 & 03 & & & & \\
\hline \multicolumn{7}{|l|}{ Estrogen receptor } \\
\hline Negative & 09 & 15 & $3.75(1.72,20.86)$ & 0.06 & $2.89(1.34,24.55)$ & 0.032 \\
\hline Positive & 09 & 04 & & & & \\
\hline \multicolumn{7}{|c|}{ Progesterone receptor } \\
\hline Negative & 08 & 14 & $3.50(1.72,18.10)$ & 0.07 & $5.41(1.62,46.84)$ & 0.012 \\
\hline Positive & 10 & 05 & & & & \\
\hline \multicolumn{7}{|l|}{ HER-2/neu } \\
\hline Negative & 14 & 08 & $4.81(1.93,26.94)$ & 0.02 & $11.25(1.47,85.94)$ & 0.019 \\
\hline Positive & 04 & 11 & & & & \\
\hline
\end{tabular}

(b)

\begin{tabular}{|c|c|c|c|c|c|c|}
\hline \multirow{2}{*}{ Parameters } & \multirow{2}{*}{ FAK High } & \multirow{2}{*}{ FAK Not High } & \multicolumn{2}{|l|}{ Univariate } & \multicolumn{2}{|l|}{ Multivariate } \\
\hline & & & Odds ratio & p-value & Odds ratio & p-value \\
\hline \multicolumn{7}{|l|}{ Age } \\
\hline Below 45 years & 11 & 08 & $2.75(1.59,13.39)$ & 0.013 & $3.19(1.58,17.49)$ & 0.018 \\
\hline 45 years and above & 06 & 12 & & & & \\
\hline \multicolumn{7}{|l|}{ Stage } \\
\hline Early & 01 & 15 & $4.80(1.28,27.04)$ & 0.00002 & $2.11(1.07,9.34)$ & 0.0041 \\
\hline Advanced & 16 & 05 & & & & \\
\hline \multicolumn{7}{|l|}{ Estrogen receptor } \\
\hline Negative & 09 & 15 & $2.67(1.54,13.81)$ & 0.016 & $0.73(0.06,7.88)$ & 0.079 \\
\hline Positive & 08 & 05 & & & & \\
\hline \multicolumn{7}{|c|}{ Progesterone receptor } \\
\hline Negative & 07 & 15 & $4.29(1.86,22.87)$ & 0.03 & $6.57(1.14,24.47)$ & 0.039 \\
\hline Positive & 10 & 05 & & & & \\
\hline \multicolumn{7}{|l|}{ HER-2/neu } \\
\hline Negative & 03 & 12 & $7.00(1.23,45.10)$ & 0.008 & $12.31(1.98$ & 0.011 \\
\hline Positive & 14 & 08 & & & $85.94)$ & \\
\hline
\end{tabular}

$\mathrm{FAK}$ high $=3$ and 4 score; FAK not high $=0,1,2$ score.

Expression of FAK may be regulated either in translational or in transcriptional stage. Our observation indicates that over production of FAK mRNA in tumor samples may be due to, either the elevation of $f a k$ gene copy number or increased promoter activity of $f a k$ gene. Early observations have shown that copy number of FAK gene is increased in tumor cells [37]. Elizabeth et al. (2008) has shown FAK mRNA expression in advanced stage of neuroblastoma. Again Lark et al. (2003) has also shown elevated FAK mRNA in colorectal cancer. A different observation by DO Watermann et al. proposed that the FAK induction is independent of primary transcriptional effects, since they did not found significant elevation of FAK transcript. We propose that FAK mRNA production is increased in tumor sample which is reflected in overexpression of FAK protein pp125.

Cance et al. proposed that over expression of FAK is an early event in breast tumorigenesis. Oktay et al. further confirmed that FAK overexpression appears to be a marker for malignant transformation of the epithelial cells but not invasive phenotype. On contrary some other groups have evidenced that FAK is over expressed in invasive tumors and may be a marker for invasive potential of a tumor [26,38].

In our study the experiments were carried with homogenates of tumor tissue. But tumor homogenate may be contaminated with non-tumor cells, and that does not show the elevation FAK expression and phosphorylation specifically in transformed cell. Considering this, our immunohistochemistry results demonstrated specific FAK overexpression and over phosphorylation (Tyr 397) in tumor cells in comparison with the surrounding adjacent non-tumor cells. These results go with previous reports on specific upregulation of FAK in tumor cells by several 
other groups [54] and also support our observations by other techniques. In our study gradual increase of specific upregulation in FAK expression and phosph orylation (Tyr 397) with the progression of tumor stage indicates towards the relevance of FAK in tumor invasiveness during advanced stages of cancer.

We compared levels of FAK with other clinicopathological features. HER-2/neu is the established marker in clinical parameters of breast cancer, which are overexpressed and usually associated with a worse prognosis. In our current study, a number of breast cancers samplesshowed HER-2/neu overexpression. The association of high FAK expression with HER-2/neu negative cases. Vadlamudi et al. proposed that HER-2/neu and heregulin are involved in FAK signaling and specifically upregulate FAK tyrosine phosphorylation. FAK was found to be involved in cell transformation and invasion mediated by ErbB-2/3 signaling [61]. Again, inhibition of Src-FAK signaling in ErbB2-positive breast cancer cells modulated focal adhesion turnover, leading to inhibition of cell invasion [62]. In our study we have found risk of upregulation of FAK expression and phosphorylation to be associated with HER2/neu negative cases. Some of the earlier observations have shown association of FAK overexpression with Her2/neu positive cases [42]. This contrast may indicate towards the difference in study population. The strong correlation of FAK expression, phosphorylation and tumor grading we found is in accordance to Schwartz and Schmitz et al.

Reports show that ER can bind to FAK and modulating FAK autophosphorylation. This observation supports the cross-talk between the ECM and the ER. This observantion also indicates towards the parallel upregulation of pression and phoshorylation were associated with ER FAK and ER [63]. In our study occurance of high levels of FAK expression was found more in ER positive and PR positive cases. Studies have also shown that progesterone induces phosphorylation of focal adhesion kinase [64]. These studies may explain the risks of FAK overexpression in ER, PR positive cases of our study. However, our observations suggest that study of FAK expression and phosphorylation in breast cancer may be considered as an indicator of development and progression of the cancer stages, and may be related with the status of different clinical parameters. Specific up- regulation of FAK expression and phosphorylation in human breast cancer suggests its important functional role in breast cancer and it may be a potential marker for breast tumor progression as well as target for therapeutic interventions.

\section{Conclusion}

FAK is involved in development and progression of breast cancer. Expression and phosphorylation of FAK is increased in tumor tissue compared to the adjacent nontumor tissue. Upregulaton of FAK is increased parrallely with progression of cancer stages, so FAK may be considered as an indicator of human breast cancer progression. Processing of FAK to lower molecular weight products is found to be increased in tumor tissue compared to the non-tumor tissue. It is also found to be increased with progression of cancer. So, FAK processing may be considered as an indicator of invasive potential of breast cancer. In breast cancer, FAK is upregulated both in protein and mRNA level. Expression and phosphorylation of FAK is increased specifically in the tumor regions compared to the surrounding non-tumor region. Localisation of FAK is membrano-cytoplasmic. Risk of FAK overexpression is more for both ER and PR positive cases compared to respective negative cases. But risk of FAK overexpression is more for Her2/neu negative cases compared to the positive cases.

\section{Acknowledgements}

The authors wish to express their thanks to Dr. Jaydip Biswas, Director, Chittaranjan National Cancer Institute, for continuous inspiration and financial support and to Defence Research \& Development Organisation (DRDO) (Grant no: DLS/81/48222/LSRB-145/ID/2008) for funding this project.

\section{REFERENCES}

[1] M. Vicente-Manzanares, D. J. Webb and A. R. Horwitz, "Cell Migration at a Glance," Journal of Cell Science, Vol. 118, No. 21, 2005, pp. 4917-4919. doi: $10.1242 /$ jes.02662

[2] S. K. Mitra, D. A. Hanson and D. D. Schlaepfer, "Focal Adhesion Kinase: In Command and Control of Cell Motility," Nature Reviews Molecular Cell Biology, Vol. 6, No. 1, 2005, pp. 56-68. doi:10.1038/nrm1549

[3] D. D. Schlaepfer and S. K. Mitra, "Multiple Connections Link FAK to Cell Motility and Invasion," Current Opinion in Genetics \& Development, Vol. 14, No. 1, 2004, pp. 92-101. doi:10.1016/j.gde.2003.12.002

[4] A. Hamadi, M. Bouali, M. Dontenwill, H. Stoeckel, K. Takeda and P. Ronde, "Regulation of Focal Adhesion Dynamics and Disassembly by Phosphorylation of FAK at Tyrosine 397," Journal of Cell Science, Vol. 118, No. 19, 2005, pp. 4415-4425. doi:10.1242/jcs.02565

[5] J. D. Owen, P. J. Ruest, D. W. Fry and S. K. Hanks, "Induced Focal Adhesion Kinase (FAK) Expression in FAKNull Cells Enhances Cell Spreading and Migration Requiring Both Auto- and Activation Loop Phosphorylation Sites and Inhibits Adhesion-Dependent Tyrosine Phosphorylation of Pyk2," Molecular and Cellular Biology, Vol. 19, No. 7, 1999, pp. 4806-4818.

[6] L. A. Cary, J. F. Chang and J. L. Guan, "Stimulation of Cell Migration by Overexpression of Focal Adhesion Kinase and Its Association with Src and Fyn," Journal of 
Cell Science, Vol. 109, No. 7, 1996, pp. 1787-1794.

[7] R. E. Gates, L. E. King Jr., S. K. Hanks and L. B. Nanney, "Potential Role for Focal Adhesion Kinase in Migrating and Proliferating Keratinocytes near Epidermal Wounds and in Culture," Cell Growth and Differentiation, Vol. 5, No. 8, 1994, pp. 891-899.

[8] D. Ilic, Y. Furuta, S. Kanazawa, N. Takeda, K. Sobue, N. Nakatsuji, S. Nomura, J. Fujimoto, M. Okada and T. Yamamoto, "Reduced Cell Motility and Enhanced Focal Adhesion Contact Formation in Cells from FAK Deficient Mice," Nature, Vol. 377, No. 6549, 1995, pp. 539-544. doi:10.1038/377539a0

[9] D. J. Sieg, C. R. Hauck and D. D. Schlaepfer, "Required role of Focal Adhesion Kinase (FAK) for Integrin-Stimulated Cell Migration," Journal of Cell Science, Vol. 112, No. 16, 1999, pp. 2677-2691.

[10] M. A. Westhoff, B. Serrels, V. J. Fincham, M. C. Frame and N. O. Carragher, "SRC-Mediated Phosphorylation of Focal Adhesion Kinase Couples Actin and Adhesion Dynamics to Survival Signaling," Molecular and Cellular Biology, Vol. 24, No. 18, 2004, pp. 8113-8133. doi:10.1128/MCB.24.18.8113-8133.2004

[11] B. van de Water, F. Houtepen, M. Huigsloot and I. B. Tijdens, "Suppression of Chemically Induced Apoptosis but Not Necrosis of Renal Proximal Tubular Epithelial (LLC-PK1) Cells by Focal Adhesion Kinase (FAK). Role of FAK in Maintaining Focal Adhesion Organization after Acuterenal Cell Injury," The Journal of Biological Chemistry, Vol. 276, No. 39, 2001, pp. 36183-36193. doi:10.1074/jbc.M102091200

[12] D. Ilic, E. A. Almeida, D. D. Schlaepfer, P. Dazin, S. Aizawa and C. H. Damsky, "Extracellular Matrix Survival Signals Transduced by Focal Adhesion Kinase Suppress p53-Mediated Apoptosis," The Journal of Cell Biology, Vol. 143 , No. 2, 1998, pp. 547-560. doi:10.1083/jcb.143.2.547

[13] S. M. Frisch, K. Vuori, E. Ruoslahti and P. Y. Chan-Hui, "Control of Adhesion-Dependent Cell Survival by Focal Adhesion Kinase," The Journal of Cell Biology, Vol. 134, No. 3, 1996, pp. 793-799. doi:10.1083/jcb.134.3.793

[14] J. E. Hungerford, M. T. Compton, M. L. Matter, B. G. Hoffstrom and C. A. Otey, "Inhibition of pp125FAK in Cultured Fibroblasts Results in Apoptosis," The Journal of Cell Biology, Vol. 135 , No. 5, 1996, pp. 1383-1390. doi:10.1083/jcb.135.5.1383

[15] L. H. Xu, X. Yang, C. A. Bradham, D. A. Brenner, A. S. Baldwin Jr., R. J. Craven and W. G. Cance, "The Focal Adhesion Kinase Suppresses Transformation-Associated, Anchorage-Independent Apoptosis in Human Breast Cancer Cells. Involvement of Death Receptor-Related Signaling Pathways," The Journal of Biological Chemistry, Vol. 275, No. 39, 2000, pp. 30597-30604. doi:10.1074/jbc.M910027199

[16] M. S. Duxbury, H. Ito, M. J. Zinner, S. W. Ashley and E. E. Whang, "Focal Adhesion Kinase Gene Silencing Promotes Anoikis and Suppresses Metastasis of Human Pancreatic Adenocarcinoma Cells," Surgery, Vol. 135, No. 5, 2004, pp. 555-562. doi:10.1016/j.surg.2003.10.017

[17] R. Braren, H. Hu, Y. H. Kim, H. E. Beggs, L. F. Reich- ardt and R. Wang, "Endothelial FAK Is Essential for Vascular Network Stability, Cell Survival, and Lamellipodial Formation," The Journal of Cell Biology, Vol. 172 , No. 1, 2006, pp. 151-162. doi:10.1083/jcb.200506184

[18] D. Wang, J. R. Grammer, C. S. Cobbs, J. E. Stewart Jr., Z. Liu, R. Rhoden, T. P. Hecker, Q. Ding and C. L. Gladson, "p125 Focal Adhesion Kinase Promotes Malignant Astrocytoma Cell Proliferation in Vivo," Journal of Cell Science, Vol. 113, No. 23, 2000, pp. 4221-4230.

[19] J. H. Zhao, H. Reiske and J. L. Guan, "Regulation of the Cell Cycle by Focal Adhesion Kinase," The Journal of Cell Biology, Vol. 143, No. 7, 1998, pp. 1997-2008. doi:10.1083/jcb.143.7.1997

[20] M. Oktay, K. K. Wary, M. Dans, R. B. Birge and F. G. Giancotti, "Integrinmediated Activation of Focal Adhesion Kinase Is Required for Signaling to Jun NH2-Terminal Kinase and Progression through the G1 Phase of the Cell Cycle," The Journal of Cell Biology, Vol. 145, No. 7, 1999, pp. 1461-1469. doi:10.1083/jcb.145.7.1461

[21] J. Zhao, R. Pestell and J. L. Guan, "Transcriptional Activation of Cyclin D1 Promoter by FAK Contributes to Cell Cycle Progression," Molecular Biology of the Cell, Vol. 12, No. 12, 2001, pp. 4066-4077.

[22] M. Toutant, A. Costa, J. M. Studler, G. Kadare, M. Carnaud and J. A. Girault, "Alternative Splicing Controls the Mechanisms of FAK Autophosphorylation," Molecular and Cell Biology, Vol. 22, No. 22, 2002, pp. 7731-7743. doi:10.1128/MCB.22.22.7731-7743.2002

[23] E. Liu, J. F. Cote and K.Vuori, "Negative Regulation of FAK Signaling by SOCS Proteins," EMBO Journal, Vol. 22, No. 19, 2003, pp. 5036-5046. doi:10.1093/emboj/cdg503

[24] N. O. Carragher, M. A. Westhoff, V. J. Fincham, M. D. Schaller and M. C. Frame, "A Novel Role for FAK as a Protease Targeting Adaptor Protein. Regulation by p42 ERK and Src," Current Biology, Vol. 13, No. 16, 2003, pp.1442-1450. doi:10.1016/S0960-9822(03)00544-X

[25] A. Katsumi, A. W. Orr, E. Tzima and M. A. Schwartz, "Integrins in Mechanotransduction," The Journal of Biological Chemistry, Vol. 279, No. 13, 2004, pp. 12001-12004. doi:10.1074/jbc.R300038200

[26] L. V. Owens, L. Xu, R. J. Craven, G. A. Dent, T. M. Weiner, L. Kornberg, E. T. Liu and W. G. Cance, "Overexpression of the Focal Adhesion Kinase (p125FAK) in Invasive Human Tumors," Cancer Research, Vol. 55, No. 13, 1995, pp. 2752-2755.

[27] X. J. Liu, L. Yang, H. B. Wu, O. Qiang, M. H. Huang and Y. P. Wang, "Apoptosis of Rat Hepatic Stellate Cells Induced by Anti-Focal Adhesion Kinase Antibody," World Journal of Gastroenterology, Vol. 8 , No. 1, 2002, pp. 734738.

[28] L. H. Xu, L. V. Owens, G. C. Sturge, X. Yang, E. T. Liu, R. J. Craven and W. G. Cance, "Attenuation of the Expression of the Focal Adhesion Kinase Induces Apoptosis in Tumor Cells," Cell Growth and Differentiation, Vol. 7, No. 4, 1996, pp. 413-418.

[29] G. Jones, J. Machado Jr., M. Tolnay and A. Merlo, "PTEN-Independent Induction of Caspase-Mediated Cell Death and Reduced Invasion by the Focal Adhesion Tar- 
geting Domain (FAT) in Human Astrocytic Brain Tumors Which Highly Express Focal Adhesion Kinase (FAK)," Cancer Research, Vol. 61, No. 15, 2001, pp. 5688-5691.

[30] S. M. Frisch, K. Vuori, E. Ruoslahti and P. Y. Chan-Hui, "Control of Adhesion-Dependent Cell Survival by Focal Adhesion Kinase," The Journal of Cell Biology, Vol. 134, No. 3, 1996, pp. 793-799. doi:10.1083/jcb.134.3.793

[31] J. E. Hungerford, M. T. Compton, M. L. Matter, B. G. Hoffstrom and C. A. Otey, "Inhibition of pp125FAK in Cultured Fibroblasts Results in Apoptosis," The Journal of Cell Biology, Vol. 135, No. 5, 1996, pp. 1383-1390. doi:10.1083/jcb.135.5.1383

[32] L. H. Xu, X. Yang, C. A. Bradham, D. A. Brenner, A. S. Baldwin Jr., R. J. Craven and W. G. Cance, "The Focal Adhesion Kinase Suppresses Transformation-Associated, Anchorage-Independent Apoptosis in Human Breast Cancer Cells. Involvement of Death Receptor-Related Signaling Pathways," The Journal of Biological Chemistry, Vol. 275, No. 39, 2000, pp. 30597-30604. doi:10.1074/jbc.M910027199

[33] M. S. Duxbury, H. Ito, M. J. Zinner, S. W. Ashley and E. E. Whang, "Focal adhesion Kinase Gene Silencing Promotes Anoikis and Suppresses Metastasis of Human Pancreatic Adenocarcinoma Cells," Surgery, Vol. 135, No. 5, 2004, pp. 555-562. doi:10.1016/i.surg.2003.10.017

[34] M. J. van Nimwegen, S. Verkoeijen, L. van Buren, D. Burg and B. van de Water, "Requirement for Focal Adhesion Kinase in the Early Phase of Mammary Adenocarcinoma Lung Metastasis Formation," Cancer Research, Vol. 65, No. 11, 2005, pp. 4698-4706.

doi:10.1158/0008-5472.CAN-04-4126

[35] S. K. Mitra, S. T. Lim, A. Chi and D. D. Schlaepfer, "Intrinsic Focal Adhesion Kinase Activity Controls Orthotopic Breast Carcinoma Metastasis via the Regulation of Urokinase Plasminogen Activator Expression in a Syngeneic Tumor Model," Oncogene, Vol. 25, No. 32, 2006, pp. 4429-4440. doi:10.1038/sj.onc. 1209482

[36] T. M.Weiner, E. T. Liu, R. J. Craven and W. G. Cance, "Expression of Growth Factor Receptors, the Focal Adhesion Kinase, and Other Tyrosine Kinases in Human Soft Tissue Tumors," Annuls of Surgical Oncology, Vol. 1, No. 1, 1994, pp.18-27. doi:10.1007/BF02303537

[37] M. Agochiya, V. G. Brunton, D. W.Owens, E. K. Parkinson, C. Paraskeva, W. N. Keith and M. C. Frame, "Increased Dosage and Amplification of the Focal Adhesion Kinase Gene in Human Cancer Cells," Oncogene, Vol. 18, No. 41, 1999, pp. 5646-5653. doi:10.1038/sj.onc. 1202957

[38] Y. Pylayeva, K. M. Gillen, W. Gerald, H. E. Beggs, L. F. Reichardt and F. G. Giancotti, "Ras- and PI3K-Dependent Breast Tumorigenesis in Mice and Humans Requires Focal Adhesion Kinase Signaling," The Journal of Clinical Investigation, Vol. 119 , No. 2, 2009, pp. 252-266.

[39] S. E. Theocharis, J. T. Klijanienko, E. Padoy, S. Athanassiou, X. X. Sastre-Garau, "Focal Adhesion Kinase (FAK) Immunocytochemical Expression in Breast Ductal Invasive Carcinoma (DIC): Correlation with Clinicopathological Parameters and Tumor Proliferative Capacity," $\mathrm{Me}$ dicine Science Monitor, Vol. 15, No. 8, 2009, pp. 221226.
[40] G. W. Cance, J. E. Harris, M. V. Iacocca, E. Roche, X. H. Yang, J. Chang, S. Simkins and L. H. Xu, "Immunohistochemical Analyses of Focal Adhesion Kinase Expression in Benign and Malignant Human Breast and Colon Tissues: Correlation with Preinvasive and Invasive Phenotypes," Clinical Cancer Research, Vol. 6, 2000, pp. 2417-2423.

[41] M. Ayaki, K. Komatsu, M. Mukai, K. Murata, M. Kameyama, S. Ishiguro, J. Miyoshi, M. Tatsuta and H. Nakamura, "Reduced Expression of Focal Adhesion Kinase in Liver Metastases Compared with Matched Primary Human Colorectal Adenocarcinomas," Clinical Cancer Research, Vol. 7, No. 10, 2001, pp. 3106-3112.

[42] K. J. Schmitz, F. Grabellus, R. Callies, F. Otterbach, J. Wohlschlaeger, B. Levkau, R. Kimmig, K. W. Schmid and H. A. Baba, "High Expression of Focal Adhesion Kinase (p125FAK) in Node-Negative Breast Cancer Is Related to Overexpression of HER-2/neu and Activated Akt Kinase but Does Not Predict Outcome," Breast Cancer Res, Vol. 7, No. 2, 2005, pp. 194-203. doi:10.1186/bcr977

[43] A. L. Lark, C. A. Livasy, L. Dressler, D. T. Moore, R. C. Millikan, J. Geradts, M. Iacocca, D. Cowan, D. Little, R. J. Craven and W. Cance, "High Focal Adhesion Kinase Expression in Invasive Breast Carcinomas Is Associated with an Aggressive Phenotype," Modern Pathology, Vol. 18, No. 10, 2005, pp. 1289-1294. doi:10.1038/modpathol.3800424

[44] S. Ménard, P. Casalini, M. Campiglio, S. M. Pupa and E. Tagliabue, "Role of HER2/neu in Tumor Progression and Therapy," Cellular and Molecular Life Science, Vol. 61, No. 23, 2004, pp. 2965-2978.

[45] B. J. Deroo and K. S. Korach, "Estrogen Receptors and Human Disease," Journal of Clinical Investment, Vol. 116, No. 3, 2006, pp. 561-567. doi:10.1172/JCI27987

[46] W. L. McGuire and G. M. Clark, "The Prognostic Role of Progesterone Receptors in Human Breast Cancer," Seminars in Oncology, Vol. 10, Supplement 4, 1983, pp. 2-6.

[47] V. Thamilselvan, D. H. Craig and M. D. Basson, "FAK Association with Multiple Signal Proteins Mediates Pressure-Induced Colon Cancer Cell Adhesion via a Src-Dependent PI3K/Akt Pathway," The FASEB Journal, Vol. 21, No. 8, 2007, pp. 1730-1741. doi:10.1096/fj.06-6545com

[48] K. Maung, D. J. Easty, S. P. Hill and D. C. Bennett, "Requirement for Focal Adhesion Kinase in Tumor Cell Adhesion," Oncogene, Vol. 18, No. 48, 1999, pp. 6824-6828. doi:10.1038/sj.onc.1203094

[49] D. D. Schlaepfer, S. K. Mitra and D. Ilic, "Control of Motile and Invasive Cell Phenotypes by Focal Adhesion Kinase," Biochimica et Biophysica Acta, Vol. 1692, No. 2-3, 2004, pp. 77-102. doi:10.1016/j.bbamcr.2004.04.008

[50] C. R. Hauck, D. A. Hsia, X. S. Puente, D. A. Cheresh and D. D. Schlaepfer, "FRNK blocks v-Src-Stimulated Invasion and Experimental Metastases without Effects on Cell Motility or Growth," The EMBO Journal, Vol. 21, No. 23, 2002, pp. 6289-6302. doi:10.1093/emboj/cdf631

[51] T. Nagy, H. Wei, T. L. Shen, X. Peng, C. C. Liang, B. Gan and J. L. Guan, "Mammary Epithelial-Specific Dele- 
tion of the Focal Adhesion Kinase Gene Leads to Severe Lobulo-Alveolar Hypoplasia and Secretory Immaturity of the Murine Mammary Gland," The Journal of Biological Chemistry, Vol. 282 , No. 43, 2007, pp. 31766-31776. doi:10.1074/jbc.M705403200

[52] V. M. Golubovskaya, F. A. Kweh and W. G. Cance, "Focal Adhesion Kinase and Cancer," Histology and Histopathology, Vol. 24, No. 4-6, 2009, pp. 503-510.

[53] J. Zhao and J. L. Guan, "Signal Transduction by Focal Adhesion Kinase in Cancer," Cancer Metastasis Reviews, Vol. 28, No. 1-2, 2009, pp. 35-49. doi:10.1007/s10555-008-9165-4

[54] D. O. Watermann, B. Gabriel, M. Jager, M. OrlowskaVolk, A. Hasenburg, A. zur Hausen, G. Gitsch and E. Stickeler, "Specific Induction of pp125 Focal Adhesion Kinase in Human Breast Cancer," British Journal of Cancer, Vol. 93 , No. 6, 2005, pp. 694-698. doi:10.1038/sj.bjc. 6602744

[55] M. H. Oktay, K. Oktay, D. Hamele-Bena, A. Buyuk and L. G. Koss, "Focal Adhesion Kinase as a Marker of Malignant Phenotype in Breast and Cervical Carcinomas," Human Pathology, Vol. 34, No. 3, 2003, pp. 240-245. doi:10.1053/hupa.2003.40

[56] B. Gabriel, A. zur Hausen, E. Stickeler, C. Dietz, G. Gitsch, D. C. Fischer, J. Bouda, C. Tempfer and A. Hasenburg, "Weak Expression of Focal Adhesion Kinase (pp125FAK) in Patients with Cervical Cancer Is Associated with Poor Disease Outcome," Clinical Cancer Research, Vol. 12, No. 8, 2006, pp. 2476-2483. doi:10.1158/1078-0432.CCR-05-1867

[57] S. K. Mitra and D. D. Schlaepfer, "Integrin-Regulated FAK-Src Signaling in Normal and Cancer Cells," Current Opinion in Cell Biology, Vol. 18, No. 5, 2006, pp. 516523. doi:10.1016/i.ceb.2006.08.011

[58] H. R. Reiske, S. C. Kao, L. A. Cary, J. L. Guan, J. F. Lai and H. C. Chen, "Requirement of Phosphatidylinositol 3-Kinase in Focal Adhesion Kinase-Promoted Cell Migration," The Journal of Biological Chemistry, Vol. 274, No. 18, 1999, pp. 12361-12366.

\footnotetext{
Abbreviations

ECM: Extracellular Matrix; FAK: Focal Adhesion Kinase, FN: Fibronectin, GAPDH: Glyceraldehyde phosphate dehydrogenase.
}

doi:10.1074/jbc.274.18.12361

[59] R. S. Sawhney, M. M. Cookson, Y. Omar, J. Hauser and M. G. Brattain, "Integrin Alpha2-Mediated ERK and Calpain Activation Play a Critical Role in Cell Adhesion and Motility via Focal Adhesion Kinase Signaling: Identification of a Novel Signaling Pathway," The Journal of Biological Chemistry, Vol. 281, No. 13, 2006, pp. 8497 8510. doi:10.1074/jbc.M600787200

[60] L. H. Xu, X. Yang, C. A. Bradham, D. A. Brenner, A. S. Baldwin Jr., R. J. Craven and W. G. Cance, "The Focal Adhesion Kinase Suppresses Transformation-Associated, Anchorage-Independent Apoptosis in Human Breast Cancer Cells. Involvement of Death Receptor-Related Signaling Pathways," The Journal of Biological Chemistry, Vol. 275, No. 39, 2000, pp. 30597-30604. doi:10.1074/jbc.M910027199

[61] N. Benlimame, Q. He, S. Jie, D. Xiao, Y. J. Xu, M. Loignon, D. D. Schlaepfer and M. A. Alaoui-Jamali, "FAK Signaling Is Critical for ErbB-2/ErbB-3 Receptor Cooperation for Oncogenic Transformation and Invasion," The Journal of Cell Biology, Vol. 171, No. 3, 2005, pp. 505516. doi:10.1083/jcb.200504124

[62] Y. Xu, N. Benlimame, J. Su, Q. He and M. A. AlaouiJamali, "Regulation of Focal Adhesion Turnover by ErbB Signalling in Invasive Breast Cancer Cells," British Journal of Cancer, Vol. 100, No. 4, 2009, pp. 633-643. doi:10.1038/sj.bjc.6604901

[63] M. D. Planas-Silva, R. D. Bruggeman, R. T. Grenko and J. Stanley Smith, "Role of c-Src and Focal Adhesion Kinase in Progression and Metastasis of Estrogen ReceptorPositive Breast Cancer," Biochemical and Biophysical Research Communications, Vol. 341, No. 1, 2006, pp. 7381. doi:10.1016/j.bbrc.2005.12.164

[64] V. C. Lin, E. H. Ng, S. E. Aw, M. G. Tan, E. H. Ng and B. H. Bay, "Progesterone Induces Focal Adhesion in Breast Cancer Cells MDA-MB-231 Transfected with Progesterone Receptor Complementary DNA," Molecular Endocrinology, Vol. 14, No. 3, 2000, pp. 348-358. doi:10.1210/me.14.3.348 